\title{
Pliable synaptic fidelity and excitatory inter-synaptic crosstalk in the intact brain
}

James P. Reynolds ${ }^{1}$, Thomas P. Jensen ${ }^{1}$, Sylvain Rama ${ }^{1}$, Kaiyu Zheng ${ }^{1}$, Leonid P. Savtchenko ${ }^{1}$, Dmitri A. Rusakov ${ }^{1}$

${ }^{1}$ UCL Queen Square Institute of Neurology, University College London, United Kingdom

Correspondence: d.rusakov@ucl.ac.uk 


\begin{abstract}
Memory formation in neural circuits may involve changes in synaptic efficacy and in cell intrinsic excitability, yet how this process unfolds in the living brain has remained elusive. Here, we employed multiplexed imaging of genetically encoded indicators of glutamate and $\mathrm{Ca}^{2+}$ in mouse barrel cortex to detect increased fidelity coupled with reduced excitation of thalamocortical connections that undergo whisker-stimulation induced LTP. High-resolution imaging revealed that whisker stimuli trigger excitatory synaptic activity that generates extrasynaptic glutamate transients reaching the bulk of neighbouring synapses in the target cortical area. Our findings pave the way to understanding basic plasticity features of the synaptic connectome while revealing a significant component of volume-transmitted glutamatergic signalling among cells in the intact brain.
\end{abstract}

\title{
One Sentence Summary
}

Sensory-stimulation LTP increases fidelity while reducing excitation at individual thalamocortical connections which generate spatially intersecting glutamate discharges 
Excitatory glutamatergic synapses underpin the workings of the brain. Their ability to change transmission strength in a coincidence-dependent manner has been considered key to the process of learning described by Hebb (1). Hebbian principles have found their dependable empirical prototype in the synaptic long-term potentiation (LTP) (2), which has since been associated with memory trace formation (3-7). However, how the build-up of synaptic potentiation evades runaway network excitation - either through homeostatic synaptic weight re-scaling (8-10), or through altered cell excitability (11-13), or both (1416) - has been disputed. The issue remains debatable because separating out synaptic efficacy changes from the effects of cell and network excitability in vivo has been difficult. In practice, LTP in the intact brain has been shown almost exclusively in the bulk of synapses, which limits our understanding of whether it involves an increase in the postsynaptic receptor current or in release probability $\left(\mathrm{P}_{\mathrm{r}}\right)$, or both.

In general, $\mathrm{P}_{\mathrm{r}}$ of small excitatory synapses can vary $>5$-fold along individual axons (17) or dendritic trees (18), and is highly use-dependent (19), whereas its average value in vivo is generally low (20). These features are in line with a theory that relates optimal information handling in the cortex predominantly to $P_{r}$ control $(21,22)$. In brain slices, direct $P_{r}$ readout can be achieved by imaging either postsynaptic $\mathrm{Ca}^{2+}$ transients $(23,24)$ or presynaptic glutamate release $(17,25)$, but such methods rely on full control of action potentials, which could be unattainable for highly interconnected in vivo networks.

We sought to address some of these long-standing issues, firstly, by establishing a $\mathrm{Pr}_{\mathrm{r}}$ tracking method that would not require spiking control in vivo, and secondly, by examining transmission at individual thalamocortical (TC) synapses in the course of LTP induced by contralateral rhythmic whisker stimulation (RWS-LTP) (26-28). To test and validate $\mathrm{P}_{\mathrm{r}}$ tracking, we took advantage of a previously established multiplexed imaging technique (17). In organotypic hippocampal slices, we patched a CA3 pyramidal cell expressing the optical glutamate sensor iGluSnFR (Fig. 1A, fig. S1A; Methods). After whole cell break-in and dialysis of the cell with the red-shifted $\mathrm{Ca}^{2+}$ indicator $\mathrm{Cal}-590$ (in some cases also with the tracer Alexa Fluor 594), we focused on individual presynaptic boutons traced along the axon, for high-speed two-photon excitation (2PE) 'Tornado scan' imaging (Fig. 1B, fig. S1B; Methods). Four action potentials evoked at the soma produced clear multiplexed readout of evoked glutamate release and presynaptic $\mathrm{Ca}^{2+}$ entry (Fig. 1C; fig. S1C). The corresponding dynamics of absolute $\mathrm{Ca}^{2+}$ concentration $\left(\left[\mathrm{Ca}^{2+}\right]\right)$ was assessed using $\mathrm{Ca}^{2+}$ sensitivity of the Cal-590 fluorescence lifetime (Fig. 1D), as detailed previously (17, 29).

In these experiments, iGluSnFR signal varied many-fold, reflecting variable quantal content (including failures) of release events (Fig. 1A-D); this variability was not due to recording instability because it was several times smaller in the matching glutamate 2PEuncaging tests (fig. S1D-F). At the same time, the spike-evoked $\mathrm{Ca}^{2+}$ signal was remarkably stable, suggesting that, in conditions of non-saturation, it would scale with the number of action potentials, a notion consistent with somatic $\mathrm{Ca}^{2+}$ imaging in vivo (30). Thus, for a given synapse, the ratio between glutamate- and $\mathrm{Ca}^{2+}$-sensitive signals (Glu/Ca) should scale with the amount of glutamate released per action potential, or synaptic release efficacy proportional to $P_{r}$. Indeed, when we plotted the Glu/Ca ratio (or 
the ratio Glu / $\left[\mathrm{Ca}^{2+}\right.$ ] entry) against direct $P_{r}$ readout (iGluSnFR signal amplitude), the relationship showed excellent linear regression (Fig. 1E).

Figure 1. The ratio of glutamate versus $\mathrm{Ca}^{2+}$ fluorescence signals at spiking presynaptic boutons can faithfully track changes in synaptic release probability $P_{r .}$ (A) CA3 pyramidal cell (organotypic

A

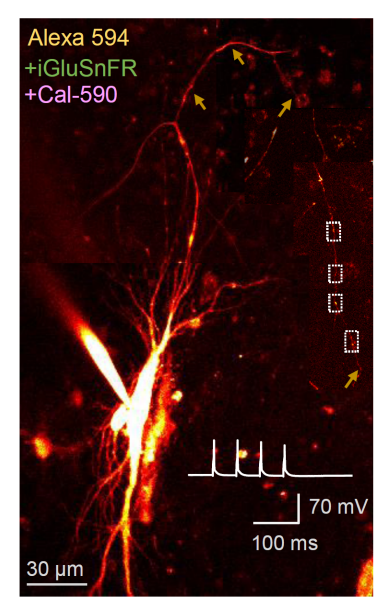

D

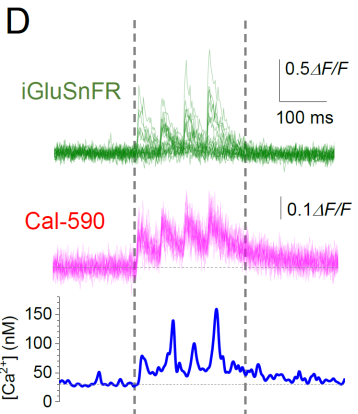

$\mathrm{E}$

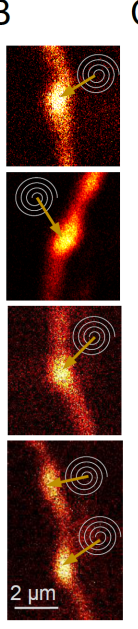

C
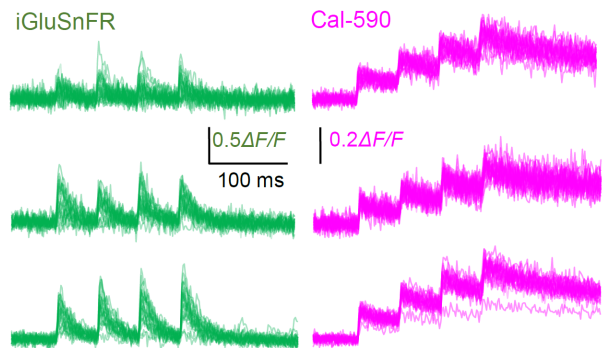

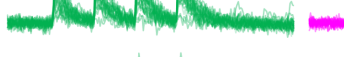
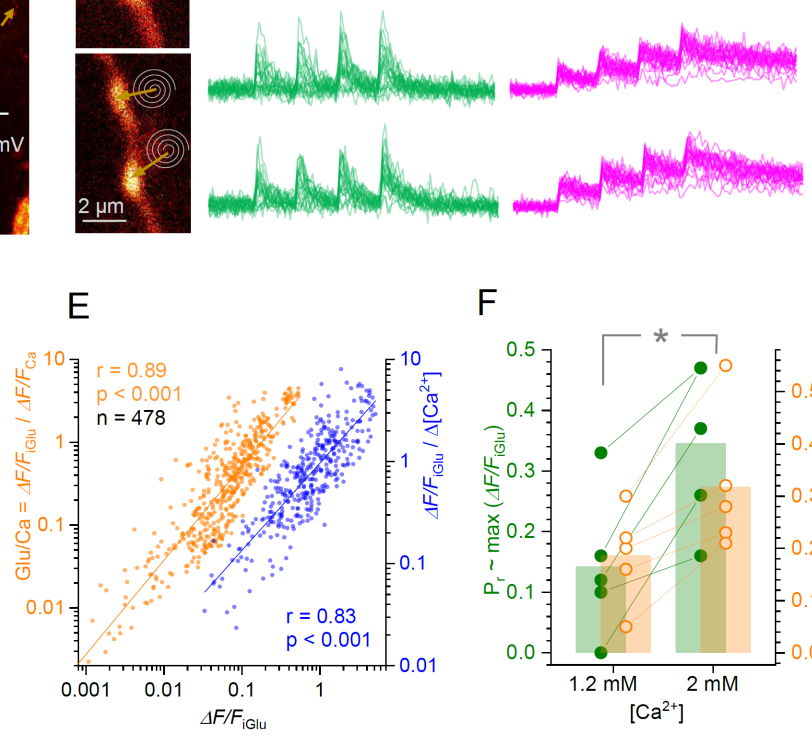

$\mathrm{F}$
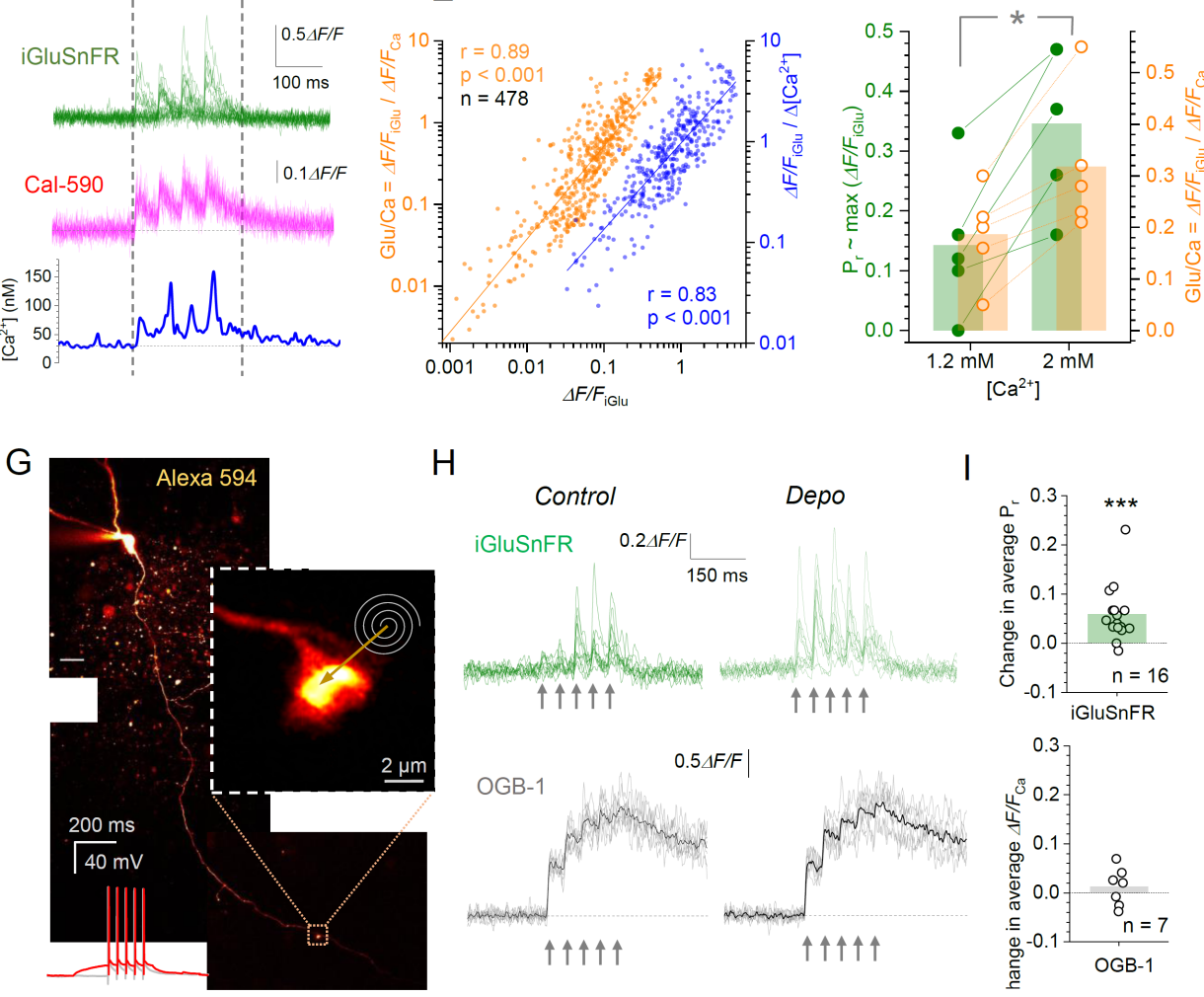

$\mathrm{H}$
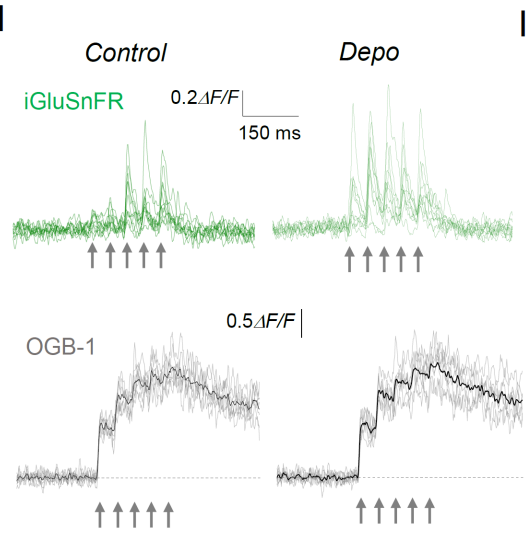

\section{।}

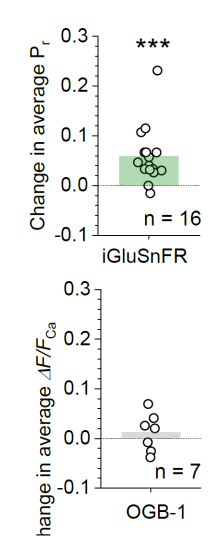

hippocampal slice)

expressing SF-

iGluSnFR.A184V and

dialysed whole-cell

with Cal-590 and $4 \mu \mathrm{M}$

Alexa-594, with 4

ROls along the axon

(arrowheads, dotted

rectangles; collage of

8-17 $\mu \mathrm{m}$ deep image

stack projections,

$\lambda_{x}{ }^{2 P}=800 \mathrm{~nm}$ for

tracing; example with

iGluSnFR tracing in

fig, S1A-B); inset

trace, four action

potentials $50 \mathrm{~ms}$ apart elicited at the soma in current-clamp. (B)

Presynaptic axonal boutons (ROls shown in A); spirals and arrows, Tornado scan positions. (C)

Fluorescence time course (integrated Tornado scan signal, $500 \mathrm{hz} \mathrm{sampling}$ (single boutons) or $333 \mathrm{~Hz}$ (multiple boutons) in iGluSnFR (green) and Cal-590 (magenta) channels $\left(\lambda_{x}{ }^{2 \mathrm{P}}=910 \mathrm{~nm}\right)$, in response to four action potentials $50 \mathrm{~ms}$ apart, as indicated, for the boutons in B; 20-24 trials shown. (D) Example of fluorescence responses as in $\mathrm{C}$ (green and magenta), with the FLIM-based readout of presynaptic $\left[\mathrm{Ca}^{2+}\right.$ (blue; bottom panel); vertical lines, sampling window for

$\Delta F / F_{0}$ value averaging. (E) The ratio of $\Delta F / F$ iGluSnFR response (250 ms over four responses, as in $D$ ) versus either $\triangle F / F$ Cal-590 response ('Glu/Ca' ratio, orange) or FLIM readout of $\left[\mathrm{Ca}^{2+}\right]$ (blue), plotted against $\triangle F / F$ iGluSnFR response (direct glutamate release readout, $n=478$ boutons); straight lines, linear regression; r, coefficient of correlation; log-log scale. (F) $\Delta F / F$ iGluSnFR (green, scales with $\mathrm{Pr}_{r}$ ) and $\mathrm{Glu} / \mathrm{Ca}$ responses (orange) at two concentrations of extracellular $\mathrm{Ca}^{2+}$, as indicated; dots, individual experiments; bars, average values; *, $p<0.01(n=5)$, in both cases. (G) Fragment of dentate gyrus granule cell dialysed whole-cell with OGB-1, with a tracked giant mossy fibre bouton (dotted rectangle; collage of 10-15 $\mu \mathrm{m}$ deep image stack projections) and Tornado scan position indicated (inset); trace, five action potentials elicited at 
the soma in current-clamp, at $V_{\mathrm{m}}=-70 \mathrm{mV}$ (grey) and during $200 \mathrm{~ms}$ depolarisation by $\sim 15 \mathrm{mV}$ (red). (H) Example of fluorescence responses in mossy fibre boutons expressing iGluSnFR (top, 11 trials shown) and those filled with OGB-1 (bottom, 10 trials; black line, average) during five action potentials (arrows), in control conditions and under somatic depolarisation, as shown by trace in $\mathrm{G}$. (I) Changes in $\mathrm{P}_{\mathrm{r}}$, as gauged by the failure rate of evoked iGluSnFR signals (top, $n=16$ boutons), and changes in presynaptic $\mathrm{Ca}^{2+}$ entry $(\Delta F / F$ OGB-1, bottom; $n=7$ boutons); dots, individual experiments; bars, average; ${ }^{* *}, p<0.001$.

To test if the Glu/Ca ratio would follow $\mathrm{P}_{\mathrm{r}}$ changes induced in the same synapse, we examined two cases, one with $\mathrm{Ca}^{2+}$-dependent and one with $\mathrm{Ca}^{2+}$-independent modulation of $\mathrm{P}_{\mathrm{r}}$. In the first case, we changed extracellular $\left[\mathrm{Ca}^{2+}\right]$ between 1.2 and $2.0 \mathrm{mM}$ and documented the corresponding change in $\mathrm{P}_{\mathrm{r}}$ at CA3-CA1 synapses using iGluSnFR readout (Fig. 1F, left ordinate). Here, Glu/Ca reliably reported $\mathrm{P}_{\mathrm{r}}$ increases in all tested synapses (Fig. 1F, right ordinate), reflecting the highly supralinear dependence of $\mathrm{P}_{\mathrm{r}}$ on $\mathrm{Ca}^{2+}$ entry hence extracellular $\left[\mathrm{Ca}^{2+}\right](31)$. We also confirmed that at $2.0 \mathrm{mM}$ extracellular $\left[\mathrm{Ca}^{2+}\right]$, Glu/Ca scaled linearly with iGluSnFR readout (fig. SG). In the second case, we monitored $\mathrm{Ca}^{2+}$ transients and glutamate release from giant mossy fibre boutons tracked from dentate granule cell somata (Fig. 1G). In this circuitry, presynaptic depolarisation can increase glutamate release (32), which appears dissociated from changes in presynaptic $\mathrm{Ca}^{2+}$ entry (33). Indeed, depolarising the soma by $\sim 15 \mathrm{mV}$ boosted glutamate release in response to five action potentials $\left(\right.$ Fig. $1 \mathrm{H}$ ) whereas $\mathrm{Ca}^{2+}$ entry remained unchanged (Fig. $1 \mathrm{I})$, at various distances from the soma (fig. $\mathrm{S} 1 \mathrm{H}$ ). These tests confirmed that, at least for relatively short spike bursts, the Glu/Ca ratio would follow the amount of glutamate released per spike, thus providing $\mathrm{P}_{\mathrm{r}}$ readout when spiking cannot be directly monitored.

Equipped with this approach, we sought to monitor $P_{r}$ changes in TC projections that respond to contralateral rhythmic whisker stimulation (RWS) in vivo. We targeted the circuitry of the posterior medial nucleus (POm) of the thalamus, monitoring axonal projections to the barrel cortex in S1 (34-36), where ramifications are widespread but sparse $(37,38)$. To enable the multiplexed imaging of presynaptic $\mathrm{Ca}^{2+}$ and glutamate release at individual connections, we virally transduced two genetically-encoded indicators, red-shifted cytosolic jRGECO1a (39) for $\mathrm{Ca}^{2+}$ and green surface-bound iGluSnFR for glutamate, in POm neurons (synapsin promoter) and S1BF astroglia (GFAP promoter), respectively (Fig. 2A), using a method established previously $(36,40)$ (Methods). Two weeks after AAV transduction, a cranial window was implanted over S1BF, so that individual presynaptic boutons of the jRGECO1a-lablled TC axons could be traced once the animal has recovered (Fig. 2A).

First, we explored the dynamic range of presynaptic $\mathrm{Ca}^{2+}$ activity in vivo, to probe saturation of jREGO1a readout. Awake animals showed spontaneous $\mathrm{Ca}^{2+}$ activity in 20$30 \%$ tentative axonal boutons that we traced through the cranial window using cytosolic jREGO1a fluorescence (Fig. 2B-C). At individual boutons, the signal amplitude was correlated with its decay (Fig. 2D; analysis detail in fig. S2A), with signal strength increasing with its frequency (fig. S2B). The latter suggested the occurrence of variableintensity spike bursts rather than uniform events. Elevating the anaesthesia level visibly suppressed spontaneous activity, by $\sim 85 \%$ (Methods), thus enabling us to record, without contamination, synaptic responses to 5 s-long RWS at $3 \mathrm{~Hz}$ (Fig. 2E). Overall, the evoked $\mathrm{Ca}^{2+}$ responses were 3-4 times stronger than individual spontaneous events, but in either 
case the average $\mathrm{Ca}^{2+}$ signal was 4-5 times smaller than the largest (Fig. 2F). This suggested, firstly, no appreciable jREGO1a signal saturation for RWS lasting 5 s or less, and secondly, that RWS-evoked responses represented multiple-spike bursts.

Figure 2. Presynaptic $\mathrm{Ca}^{2+}$ imaging in thalamocortical (TC) axonal boutons covers the dynamic range of axonal firing enabling Pr tracking in vivo using the Glu/Ca ratio. (A) Experimental arrangement

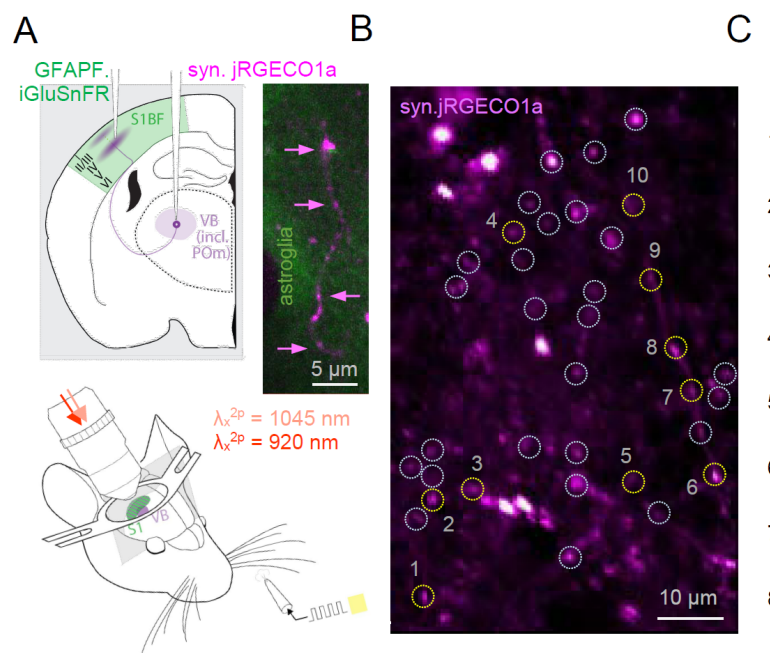

E

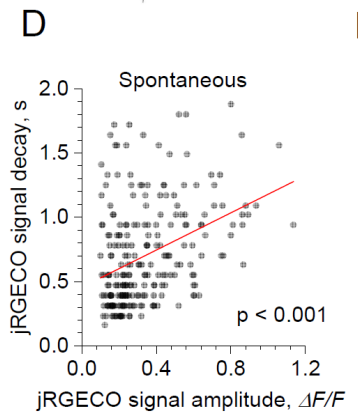

G

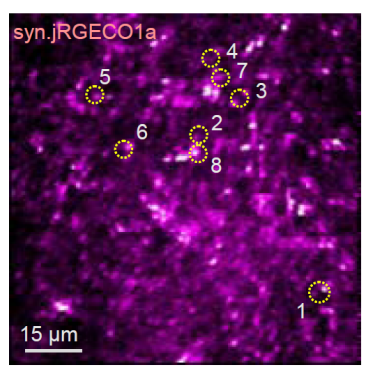

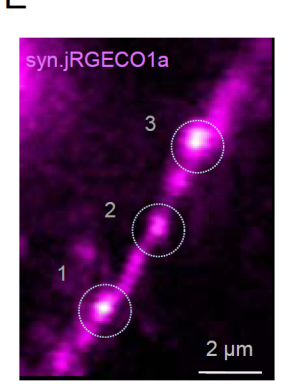

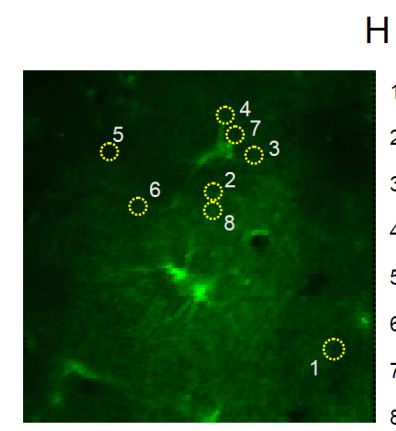

C

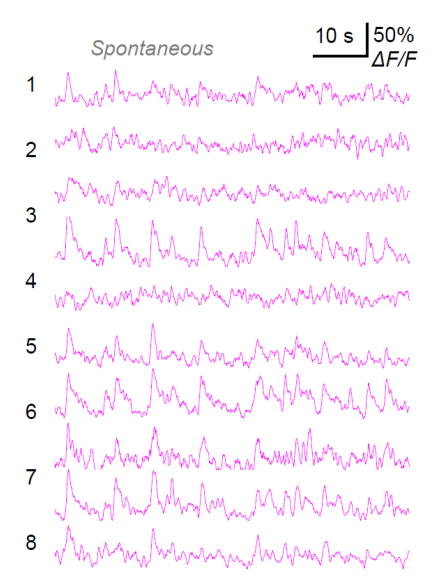

$\mathrm{F}$ illustrating viral transduction of jRGECO1a and iGluSnFR.A184S in the barrel cortex S1BF astroglia, and thalamus VB neurons, respectively (top); with a cranial window, under dual length two-photon excitation regime, as illustrated (bottom); inset image, snapshot through the cranial window depicting expression of jRGECO1a in TC axons (magenta, arrowheads) and iGluSnFR in local astroglia (green); see Methods. (B) A widerframe snapshot (jRGECO1a channel; depth $\sim 100 \mu \mathrm{m}$, layer 23 S1BF) showing tentative TC axonal boutons (selected arbitrarily, based on morphology; dotted circles). (C) Snapshot of spontaneous $\mathrm{Ca}^{2+}$ activity recorded in numbered boutons shown in B (other candidate boutons appeared silent). (D) The decay of spontaneous presynaptic $\mathrm{Ca}^{2+}$ signals recorded as in B-C, plotted against the $\Delta F / F_{0}$ signal amplitude (jRGECO1a fluorescence, $n=233$

events); line, linear regression (significant slope at $p<0.001)$. (E) An example of TC axonal fragment with three jRGECO1a-expressing boutons (1-3, left) and their fluorescence responses to a contralateral rhythmic whisker stimulation (RWS, $5 \mathrm{~s}$ at $3 \mathrm{~Hz}$; grey segment, right). (F) Frequency distribution of the presynaptic signal strength (area under the $\triangle F / F 0$ curve) for spontaneous and evoked events at TC boutons, as indicated. (G) Example of multiplexed-imaging snapshots in the jRGECO1a (left) and iGluSnFR (right) channels, with tentative TC axonal boutons (dotted circular ROls) selected based on the detectable presynaptic $\mathrm{Ca}^{2+}$ and glutamate release activity. $(\mathbf{H})$ Fluorescence responses in TC axonal boutons shown in G (area-integrated in ROls 1-8 in G) to contralateral RWS (5 s at $3 \mathrm{~Hz}$, grey area), as indicated.

We therefore switched to multiplexed imaging mode (Fig. 2G), detecting RWS-evoked glutamate release in some (20-30\%) of the TC axonal boutons that show $\mathrm{Ca}^{2+}$ activity (Fig. $2 \mathrm{H}$ ). Indeed, multiple-trial recordings showed much lower failure rates for $\mathrm{Ca}^{2+}$ compared 
with glutamate signal (fig. S2C-E), consistent with the stochastic nature of its release and/or detection failure due to the prevalence of glutamate uptake (see below). We next focused on individual boutons using Tornado scan regime and documented RWS-evoked responses in $\mathrm{Ca}^{2+}$ and glutamate channels (Fig. 3A-B), in control conditions and 10-30 min following the RWS-LTP induction protocol (5 Hz for 120s; Fig. 2C). In individual boutons, LTP induction was followed by a significant increase in the Glu/Ca ratio (by $47 \pm 12 \%$, p < $0.001, n=19$ boutons in 6 animals; Fig. 3D, fig. S3A-B), reflecting the increased average synaptic $P_{r}$. Consistent with optical quantal-analysis studies in slices (24), the smallest $P_{r}$ readout was correlated with the biggest change post-LTP (Fig. 3E).

Figure 3. Increased fidelity and decreased excitability of TC synapses following rhythmic-whiskerstimulation induced long-term potentiation (RWS-LTP). (A) A snapshot of multiplexed frame imaging,
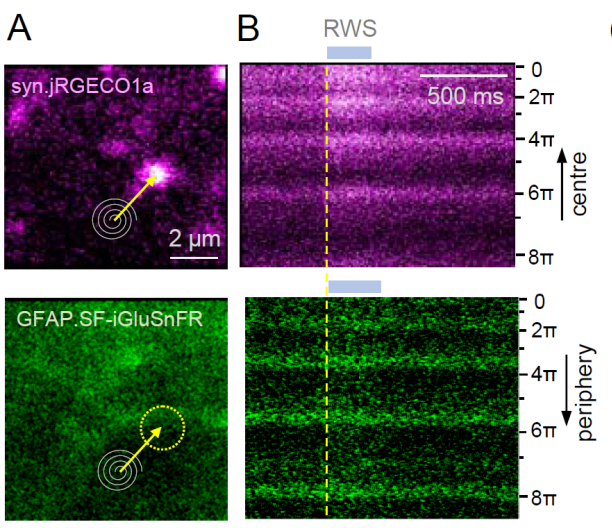

D

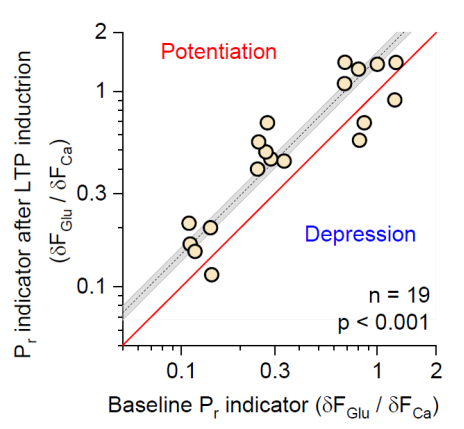

E

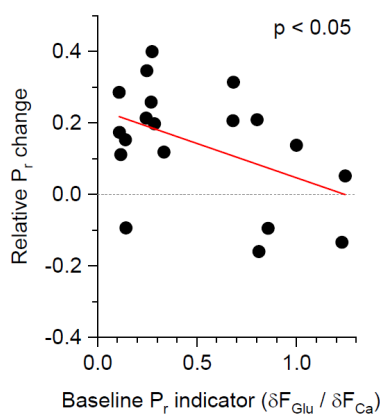

G
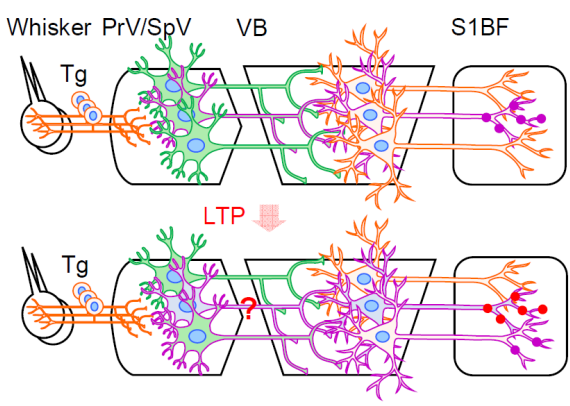

F

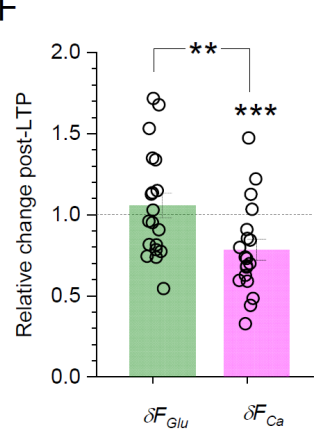

C

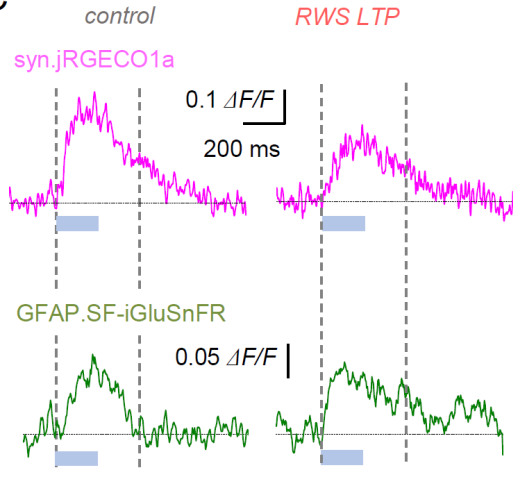

$\mathrm{H}$

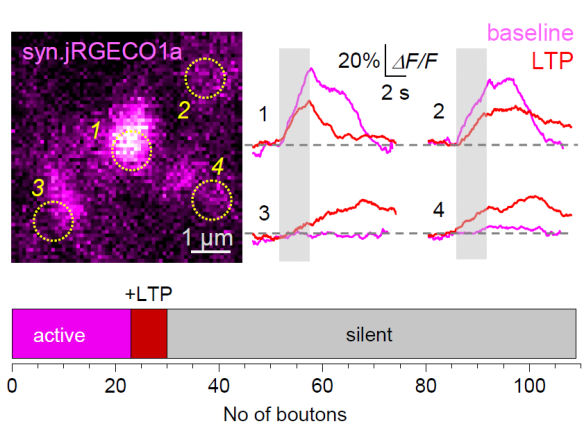
with a jRGECO1aexpressing TC axonal bouton (top, magenta) and local iGluSnFRexpressing astroglia (bottom, green; dotted circle, bouton of interest ROI); spirals and arrows, Tornado scan position. (B) Tornado scan example for the bouton in $A$, showing a fluorescent transient upon 200 ms RWS (grey segment; dotted line, onset) in both channels; ordinate, rotation angle of the Tornado spiral (rad). (C) Boutonaverage (Tornado-scan integrated, $\sim 500 \mathrm{~Hz}$ sampling) fluorescence time course in jRGECO1a and iGluSnFR channels, as indicated, during $200 \mathrm{~ms}$ RWS stimulation (grey segment), before (left) and 10-30 min after RWS-LTP induction protocol (right); average of four trials. Dotted lines, sampling windows to calculate the $\mathrm{Glu} / \mathrm{Ca}$ ratio (using areas under the $\Delta F / F_{0}$ curves). (D) Statistical summary of experiments shown in AC: Glu/Ca ratio $10-30$ min after LTP induction plotted against Glu/Ca ratio in control condition; solid line, no-change boundary; dots, individual boutons ( $n=19$ boutons in 6 animals); dotted line and grey segment, mean \pm SEM; log-log scale; $p<0.001, H_{0}$ hypothesis (no change) rejection level, one-sample t-test; further data presentation in fig. S3A-B. (E) Relative change in Glu/Ca ratio upon RWS-LTP induction, plotted against 
baseline $\mathrm{Glu} / \mathrm{Ca}$, dataset as in $\mathrm{D}$; solid line, linear regression (slope, $\mathrm{p}<0.05)$. (F) Relative changes, upon RWS-LTP, in the bouton-average $\Delta F / F_{0}$ responses recorded in the iGluSnFR (green) and jRGECO1a (magenta) channels, as in C; dots, individual boutons $(n=19)$, bars, mean \pm SEM; ${ }^{* *}, p<0.01 ;{ }^{* * *}, p<$ 0.005. (G) Diagram of neural circuitry that could be activated by whisker stimulation, in control conditions (top) and after RWS-LTP induction (bottom). Notations: magenta dots and cell boundaries, activated cells and synapses; PrV/SpV, principal and spinal nuclei of the trigeminal complex (brainstem); POm, posterior medial nucleus of the thalamus); S1BF (barrel cortex layers 2-3). (H) Image, example of four arbitrarily selected TC axonal bouton (jRGECO channel); traces: boutons 1 and 2 show robust $\mathrm{Ca}^{2+}$ response to RWS (grey segment), which is reduced 10-20 min after RWS-LTP; boutons 3 and 4 show $\mathrm{Ca}^{2+}$ response after but not before RWS-LTP induction; graph, summary: among arbitrarily selected tentative TC boutons 96 were $\mathrm{Ca}^{2+}$-silent throughout (grey), 23 were active before (magenta) and additional 7 (red) after RWS-LTP induction (data from 3 animals).

Surprisingly, the Glu/Ca ratio increase was mainly due to a decrease in the presynaptic $\mathrm{Ca}^{2+}$ signal rather than an increase in glutamate transients (Fig. 3F). This was not because of the differential photobleaching of jRGECO1a versus iGluSnFR: both channels revealed a similarly small $(\sim 12 \%)$ decrease in the baseline fluorescence post-induction (fig. S3C). Thus, the data appeared to suggest that RWS-LTP induction lowered excitability of the projecting VB neurons, or possibly of the upstream primary trigeminal nucleus neurons, or both, while providing their unchanged excitatory drive (the amount of glutamate discharged) in the barrel cortex for the same RWS (Fig. 3G). However, RWS-LTP is known to boost postsynaptic responses in cortical neurons $(26,28)$. We therefore hypothesised that RWS-LTP, while reducing excitability of the active pathway, increases excitability of neuronal projections that were silent in control conditions (Fig. 3G). In this case, we should be able to detect an increase in the numbers of $\mathrm{Ca}^{2+}$-active axonal boutons after LTP induction. We therefore monitored $\mathrm{Ca}^{2+}$ activity in 119 tentative axonal boutons (randomly selected, by their morphology only), and found that 23 were active in control conditions whereas additional 7 became active post-LTP (Fig. $3 \mathrm{H})$. This observation suggests that there could be a redistribution of excitability among cortical networks during RWS-induced plasticity, a phenomenon predicted earlier based on current-source density mapping (26).

Indeed, modulation of intrinsic cell excitability has emerged as an important constituent of memory trace formation $(12,14,41-44)$, and several mechanisms have been reported which underpin activity-dependent bidirectional excitability changes in cortical neurons (45, 46). The decreased intensity of evoked synaptic discharges at active TC synapses during LTP found here (Fig. 3F) could reflect the inverse relationship between recent cell firing activity and its excitability, possibly via regulation of the $I_{h}$ current $(41,47)$ or $\mathrm{K}^{+}$ conductance $(48,49)$, as shown in various neuronal types. Conversely, the LTP-related increase in the number of active TC synapses (Fig. $3 \mathrm{H}$ ) could potentially be explained by the increased excitability of thalamic neurons projecting to the cortex (Fig. 3G) after repetitive subthreshold activation of their glutamate inputs, as found in other settings (50$52)$.

It has recently transpired that, in similar experimental settings, LTP could drive glutamate transporter expressed by local astroglia away from potentiated synapses (40). Although this appears to boost extrasynaptic glutamate escape in ex vivo preparations $(40,53)$, little is known about extrasynaptic glutamate escape in the intact brain. The notion of 
glutamate-mediated synaptic crosstalk among central synapses has been contentious, despite evidence in brain slices (54-58), because synaptic connectivity is traditionally considered as 'wired' circuitry, akin to that in computer chips.

Figure 4. Extrasynaptic glutamate signalling in TC axonal boutons in response to whisker stimulation. (A) A snapshot of high-resolution Tornado-scan multiplexed imaging, showing a jRGECO1a-

A

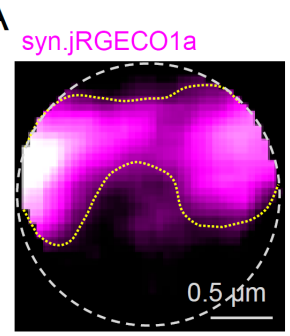

GFAP.SF-iGluSnFR
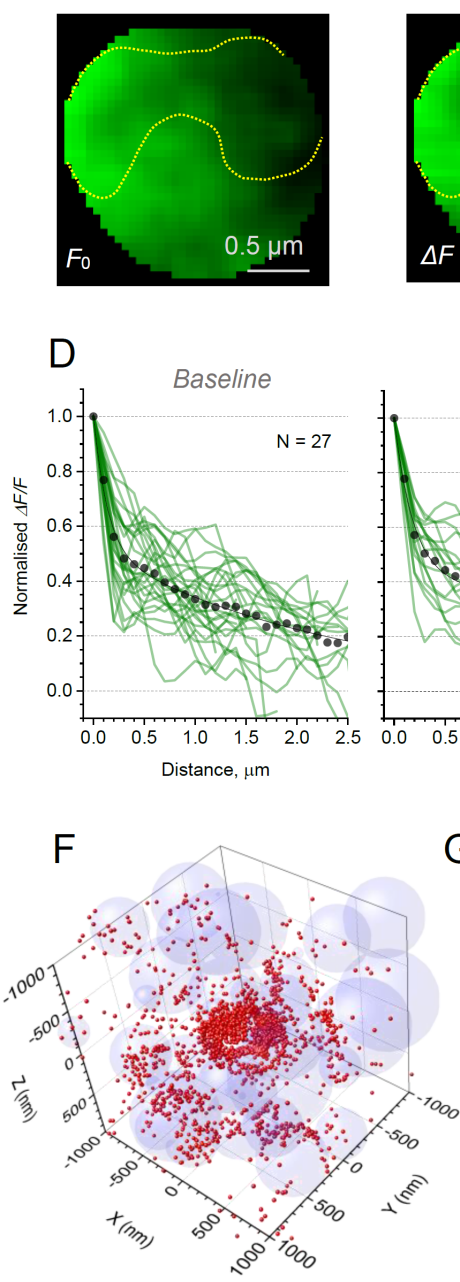

B iGluSnFR
jRGECO1a
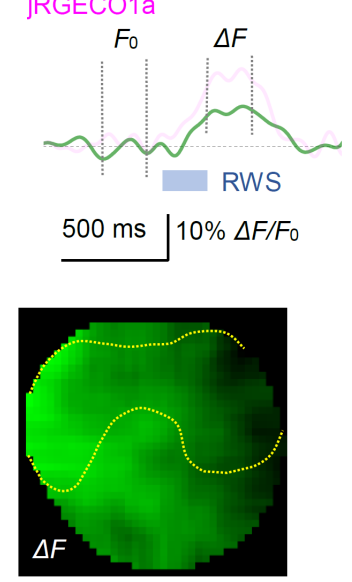

E

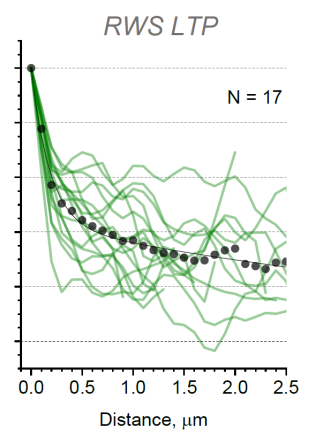

G

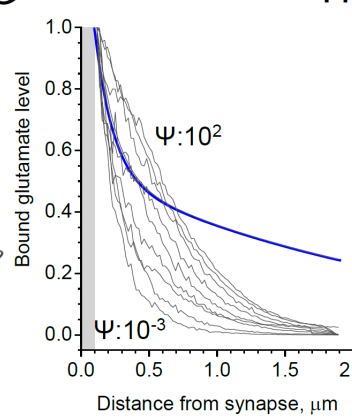

C

$\mathrm{H}$
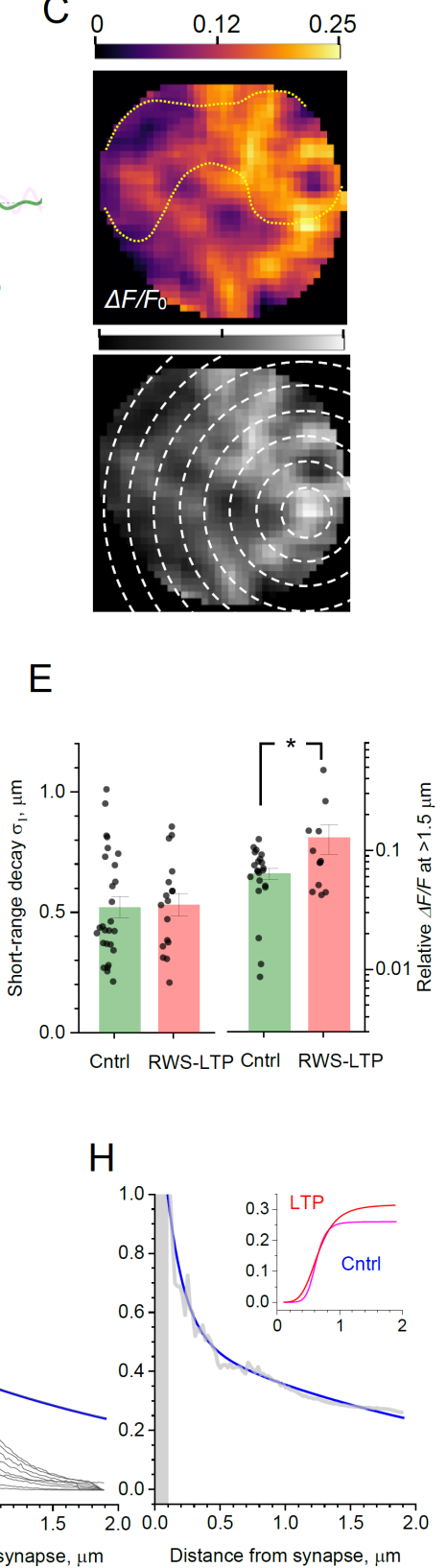

expressing TC axonal bouton (top, magenta) and the surrounding iGluSnFRexpressing astroglia (bottom, green), in resting conditions $(200 \mathrm{~ms}$ average at $500 \mathrm{~Hz}$ sampling); dotted circle, Tornado scan area coverage; yellow dotted line, axonal bouton morphology outline as judged by the fluorescence of cytosolic jRGECO1a. (B) Traces, Tornado scanintegrated fluorescence time course in the iGluSnFR channel (green; jRGECO1a magenta channel shown for illustration only), during 200 ms RWS stimulation (grey segment); vertical dotted lines, sampling windows (resting and signal intervals) for generating the $\Delta F / F_{0}$ iGluSnFR signal landscape. Image (bottom), landscape of iGluSnFR fluorescence as in A, but during the evoked signal (200 ms average at $500 \mathrm{~Hz}$ sampling). (C) Top, the $\triangle F / F_{0}$ iGluSnFR signal landscape generated as the ratio of $\Delta F$ image in $B$ and $F_{0}$ Image in $A$; bar, false colour signal scale; arrow, hotspot (fluorescence peak). Bottom, image as above, but in calibrated grey scale, with concentric circles to calculate average signal decay from the hotspot

(further examples in fig. S4A). (D) Normalised decay profile of the $\Delta F / F_{0}$ iGluSnFR signal, with respect to the hotspot, in baseline conditions (left) and 10-30min after RWS-LTP induction (right); green lines, individual axonal boutons; dots, average data; solid line, best-fit biexponential approximation in the form $A_{1} \cdot \exp \left(-\mathrm{x} / \lambda_{1}\right)+$ $A_{2} \cdot \exp \left(-\mathrm{x} / \lambda_{2}\right)$; control best-fit shown: $A_{1}=0.491, \lambda_{1}=0.134 \mu \mathrm{m}, A_{2}=0.518, \lambda_{2}=2.38 \mu \mathrm{m} ; \mathrm{LTP}$ best-fit: $A_{1}=$ $0.572, \lambda_{1}=0.181 \mu \mathrm{m}, A_{2}=0.431, \lambda_{2}=5.42 \mu \mathrm{m}$. (E) Short-range decay constant (left) and the average $\Delta F / F_{0}$ iGluSnFR signal value for distances $>1.5 \mu \mathrm{m}$ form the hotspot, compared for control conditions and postLTP, as indicated; dots, individual bouton data; bars, mean \pm SEM; ${ }^{*}, p=0.024$. (F) Simulation snapshot (2 $\mu \mathrm{m}$ wide fragment of the $4 \mu \mathrm{m}$ simulation arena) of release, diffusion, and binding of glutamate molecules 
(red dots) to astroglia-expressed iGluSnFR (and glutamate transporters GLT-1), in the neuropil modelled in silico by a scatter of overlapping spheres (text and Methods); for clarity, only spheroid shapes representing astroglia are shown (see fig. S4C-D for all-component illustrations); free diffusivity $D=0.5 \mu \mathrm{m}^{2} / \mathrm{ms}$, glutamate-indicator binding efficiency parameter (Methods) $\Psi=0.1 \mathrm{~ms}$; time point, $4 \mathrm{~ms}$ post-release. (G) Black lines, simulated concentration profiles of iGluSnFR-bound glutamate, relative to the release site (grey segment, synaptic cleft area), over the range of $\Psi(0.001,0.01,0.1,0.3,0.5,0.75,1,10,100 \mathrm{~ms})$; lowest and highest $\Psi$ values shown; blue line, experimental profile of the $\Delta F / F_{0}$ iGluSnFR signal, as in D (control conditions). (H) Experimental profile as in $\mathrm{G}$ (blue, main graph), with the best-fit simulated profile (grey) at binding efficiency $\Psi=0.5 \mathrm{~ms}$ in the initial part $(<0.7 \mu \mathrm{m})$, and a best-fit tail addition (at $>0.7 \mu \mathrm{m})$ shown in the inset graph, in control condition (blue) and after RWS-LTP (red).

To address this, we imaged jRGECO1a-filled axonal boutons using 2D reconstructions of Tornado scans (17) (Fig. 4A, top) while recording landscapes of iGluSnFR fluorescence in the surrounding astroglia (Fig. 4A, bottom). The iGluSnFR landscapes were averaged over two 200 ms time windows, one before and one during the RWS-induced response (Fig. 4B). The numerical ratio between the two corresponding images thus provided a $2 \mathrm{D}$ landscape of $\Delta F / F_{O}$ readout reflecting the extracellular $2 \mathrm{D}$ glutamate transient profile, at and near the synapse (Fig. $4 \mathrm{C}$, top). In most cases, the $\Delta F / F_{0}$ image revealed a hotspot, pointing to the tentative glutamate release site, with the signal fading with distance (Fig. 4; fig. S4A). The analysis of these images suggested, firstly, that in control conditions the RWS-evoked glutamate transient extends for at least $\sim 2.5 \mu \mathrm{m}$ from the release site, with a biexponential decay (Fig. 4D, left). Secondly, that RWS-LTP induction does not change its decay profile within $\sim 0.5 \mu \mathrm{m}$ from the release site while increasing significantly its longerrange component (at $>1.5 \mu \mathrm{m}, \mathrm{p}<0.02$; Fig. 4D-E).

In this brain region, the volume density of cortical-cortical (CC) and thalamocortical (TC) synapses is, respectively, $\sim 1.10$ and $\sim 0.23 \mu \mathrm{m}^{-3}(59,60)$, giving a random-scatter average nearest-neighbour distance (61) of $0.90 \mu \mathrm{m}$ between TC synapses, and $0.53 \mu \mathrm{m}$ between any synapses. Thus, the average $\Delta F / F_{0}$ decay profile (Fig. 4D), which reflects glutamate bound to iGluSnFR post-release, points to significant inter-synaptic crosstalk involving glutamate receptors with the affinity comparable with iGluSnFR or higher. To understand whether such crosstalk can be generated by an individual (recorded) synapse or whether it reflects multiple synaptic activation in the local area, we modelled a $4 \mu \mathrm{m}$ cube of the barrel cortex neuropil, by expanding a previously established Monte Carlo approach (62, 63). The tissue was modelled using a random scatter of unequal overlapping spheres (64) that would represent cortical astroglia taking $\sim 10 \%$ tissue volume fraction (65) and neuronal structures taking $\sim 70 \%$, leaving free $\sim 20 \%$ volume (space filling $\beta=0.8$ ) for the extracellular space $(66,67)$ (Fig. 4F; Methods).

Glutamate molecules were 'released' inside a $220 \mathrm{~nm}$-wide, $20 \mathrm{~nm}$-high synaptic cleft (59), diffusing with $D=0.5 \mu \mathrm{m}^{2} / \mathrm{ms}$ measured previously with anisotropy-FLIM (68). First, we validated the approach by comparing the outcome of Monte Carlo simulations of freely diffusing molecules with the theoretical value of Maxwell's diffusivity $\left(0.09 \mu \mathrm{m}^{2} / \mathrm{ms}\right.$ for $\beta=$ 0.8 ) in a similar porous medium (Fig. 4B). Next, we controlled the binding of molecules to the astroglia-representing spheroid shapes using time constant (integrated parameter) $\Psi$ that represents the unknown local concentration of glutamate-binding entities, such as iGluSnFR and the main glial glutamate transporter GLT-1 $(69,70)$ (Fig. 4F, fig. S4C-D; Methods). Thus, $\Psi$ was the only free parameter to fit the simulated distribution of bound 
particles to the experimental $\Delta F / F_{0}$ iGluSnFR profile (shown in Fig. 4D). Intriguingly, varying $\Psi$ value $10^{6}$-fold (from no binding to $100 \%$ instantaneous binding) consistently predicted a shorter glutamate diffusion spread than seen experimentally (Fig. 4G). The consistent mismatch suggested that glutamate signal at $>0.5 \mu \mathrm{m}$ from the synapse should come mainly from neighbouring sources. This missing signal 'tail' was well approximated by a Hill function, showing a $20 \%$ increase after LTP induction (Fig. $4 \mathrm{H}$ ).

These data suggest that, in the barrel cortex, RWS triggers synaptic glutamate discharges that reach the bulk of local synapses. This appears consistent with the observation in acute slices that even mild activation of afferent fibres generates local iGluSnFR signals with no detectable release failures (18) that are otherwise prominent when firing is limited to just one presynaptic cell (17) (Fig. 1A-D). In fact, the $\Delta F / F_{0}$ iGluSnFR landscapes obtained here (Fig. 4C, fig. S4A) display virtually no 'negative' signal, suggesting a contiguous RWS-induced glutamate rise over at least 2-3 $\mu \mathrm{m}$ wide areas. Whether this occurs due to activation of TC synapses only or whether it also involves raised activity in local circuitry remains to be established.

Because the evoked $\Delta F / F_{0}$ iGluSnFR signal reflects glutamate that binds rapidly to the indicator, assessing receptor actions of free-diffusing glutamate is not straightforward. Firstly, astroglia-expressed iGluSnFR will compete for glutamate with high-affinity glial glutamate transporters, mainly $\operatorname{GLT}-1$ type $(53,70)$. Because the on-rates of glutamate binding to iGluSnFR and GLT-1 are comparable $(71,72)$, and because both iGluSnFR and GLT-1 are co-expressed by astroglia, glutamate will bind to either in proportion to their local concentrations (cell-surface densities). Thus, the low local iGluSnFR/GLT-1 expression ratio could prevent detection of glutamate release by iGluSnFR, which is a possible reason why we found multiple TC synapses showing $\mathrm{Ca}^{2+}$ activity but no glutamate signal. By the same token, a dense patch of high-affinity receptors (such as NMDA or mGluR type) could be activated by glutamate at distances shown by the iGluSnFR detection. The prevalence and role of such volume-transmitted glutamatergic signals remain an open question. A significant reduction of local glutamate uptake capacity, either by withdrawal of perisynaptic astrocytic process (40) or localised activitydependent inhibition of GLT-1 (73), could clearly boost such phenomena and a significant reduction of local glutamate uptake capacity could clearly boost such phenomena (40), which might play an important role in regulating cognitive functions of the human brain (74, 75).

\section{METHODS}

\section{Organotypic Slice Preparation}

Organotypic hippocampal slice cultures were prepared and grown with modifications to the interface culture method (76) from P6-8 Sprague-Dawley rats, in accordance with the European Commission Directive (86/609/EEC) and the United Kingdom Home Office (Scientific Procedures) Act (1986). Three hundred $\mu \mathrm{m}$ thick, isolated hippocampal brain slices were sectioned using a Leica VT1200S vibrotome in ice-cold sterile slicing solution consisting (in $\mathrm{mM}$ ) of Sucrose 105, $\mathrm{NaCl} 50, \mathrm{KCl} 2.5, \mathrm{NaH} 2 \mathrm{PO} 4$ 1.25, $\mathrm{MgCl} 27, \mathrm{CaCl} 20.5$, Ascorbic acid 
1.3, Sodium pyruvate 3, NaHCO3 26 and Glucose 10. Following washes in culture media consisting of 50\% Minimal Essential Media, 25\% Horse Serum, 25\% Hanks Balanced Salt solution, $0.5 \%$ L-Glutamine, $28 \mathrm{mM}$ Glucose and the antibiotics penicillin $(100 \mathrm{U} / \mathrm{ml})$ and streptomycin $(100 \mu \mathrm{g} / \mathrm{ml})$, three to four slices were transferred onto each $0.4 \mu \mathrm{m}$ pore membrane insert (Millicell-CM, Millipore, UK), kept at $37^{\circ} \mathrm{C}$ in $5 \% \mathrm{CO} 2$ and fed by medium exchange for a maximum of 21 days in vitro (DIV).

\section{Biolistic Transfection of iGluSnFR variants}

The second generation iGluSnFR variant SF-iGluSnFR.A184V, kindly gifted by Prof. Loren Looger was expressed in CA3 pyramidal cells in organotypic slice cultures using biolistic transfection techniques adapted from manafacturer's instructions. In brief, $6.25 \mathrm{mg}$ of 1.6 $\mu \mathrm{m}$ Gold microcarriers were coated with $30 \mu \mathrm{g}$ of SF-iGluSnFR.A184V plasmid.

Organotypic slice cultures at 5DIV were treated with culture media containing $5 \mu \mathrm{M}$ Ara-C overnight to reduce glial reaction following transfection. The next day cultures were shot using the Helios gene-gun system (Bio-Rad) at 120psi. The slices were then returned to standard culture media the next day and remained for 5-10 days before experiments were carried out.

\section{Axon tracing and 2PE imaging in axonal boutons}

We used a Femtonics Femto2D-FLIM or a Femto3D-RC imaging system, integrated with patchclamp electrophysiology (Femtonics, Budapest) and linked on the same light path to two femtosecond pulse lasers MaiTai (SpectraPhysics-Newport) with independent shutter and intensity control. Patch pipettes were prepared with thin walled borosilicate glass capillaries (GC150-TF, Harvard apparatus) with open tip resistances 2.5-3.5 MOhm. For CA3 pyramidal cells and dentate granule cells, the internal solution contained (in mM) 135 potassium methanesulfonate, 10 HEPES, 10 di-Tris-Phosphocreatine, $4 \mathrm{MgCl}_{2}$, $4 \mathrm{Na}_{2}$-ATP, $0.4 \mathrm{Na}-\mathrm{GTP}(\mathrm{pH}$ adjusted to 7.2 using $\mathrm{KOH}$, osmolarity 290-295), and supplemented with Cal-590 (300 $\mu$ M; AAT Bioquest) for FLIM imaging in CA3 pyramidal cell axons.

Presynaptic imaging at CA3-CA1 synapses was carried out using an adaptation of presynaptic glutamate and $\mathrm{Ca}^{2+}$ imaging methods previously described (17). CA3 pyramidal cells were first identified as iGluSnFR expressing using 2PE imaging at $910 \mathrm{~nm}$ and patched in whole cell mode as above. Following break-in, 30-45 minutes were allowed for Cal-590 to equilibrate across the axonal arbour. Axons, identified by their smooth morphology and often torturous trajectory were followed in frame scan mode to their targets and discrete boutons were identified by criteria previously demonstrated to reliably match synaptophysin labelled punctae (77). In some cases, $4 \mu \mathrm{M}$ Alexa 594 was included with Cal-590 in the internal solution. The distinct two-photon excitation profiles of these two red emitting dyes enable morphology to be traced by Alexa 594 emission with $800 \mathrm{~nm}$ excitation (Fig. 1A-B), and $\mathrm{Ca}^{2+}$ signals to be recorded in the same structure by Cal-590 emission at $910 \mathrm{~nm}$ excitation (Fig. 1C), with no significant contribution of Alexa fluorescence to the Cal-590 emission.

To image glutamate release from mossy fibre giant boutons, dentate gyrus granule cells expressing iGluSnFR (using biolistic transfection, as described above) were patched, the axons were followed until putative giant boutons (main input to CA3 pyramidal cells) were identified as varicosities on axon collaterals with a width of at least 2-3 $\mu \mathrm{m}$ and one or more visible filopodial 
protrusions $(78,79)$. In separate experiments, granule cells were dialysed with the $\mathrm{Ca}^{2+}$ indicator OGB-1 $(200 \mu \mathrm{M})$.

For fast imaging of action-potential evoked iGluSnFR, Cal-590, or OGB-1 fluorescence transients at single boutons, a spiral shaped ("Tornado") scan line was placed over the bouton of interest (described further in the text) which was then scanned at a sampling frequency of $\sim 500 \mathrm{~Hz}$ with excitation at $910 \mathrm{~nm}$. For multi-bouton imaging point-scans were made with a temporal resolution 333 or $250 \mathrm{~Hz}$, usage described further in the text. Following a baseline period, action potentials initiated by brief positive voltage steps in voltage clamp mode $\left(\mathrm{V}_{\mathrm{m}}\right.$ holding $\left.-70 \mathrm{mV}\right)$ were given with an interval of $50 \mathrm{~ms}$.

\section{Cal-590 FLIM readout of $\mathrm{Ca}^{2+}$ concentration}

Using the scanning methodologies described above line-scan data were recorded by both standard analogue integration in Femtonics MES and in TCSPC in Becker and Hickl SPCM using dual HPM-100 hybrid detectors. FLIM line scan data were collected as previously described (17, 68 ) and stored as $5 \mathrm{D}$-tensors $(\mathrm{t}, \mathrm{x}, \mathrm{y}, \mathrm{z}, \mathrm{T})$ to be analysed with custom written data analysis software available at (https://github.com/zhengkaiyu/FIMAS). Since morphological data was not of value when scanning only within the bouton $x, y$ and $z$ data were summed along their respective axes. For measurements of single trial baseline and action potential $\left[\mathrm{Ca}^{2+}\right]$ data were then summed along the time axis with bin sizes adequate for accurate the use of the NTC measure to extrapolate $\left[\mathrm{Ca}^{2+}\right]$ from FLIM data. multiple trials were summed to produce photon counts high enough to accurately determine dynamics of single action potential $\left[\mathrm{Ca}^{2+}\right]$ transients.

\section{Imaging Uncaging evoked glutamate transients at single pre-synaptic boutons}

Uncaging evoked glutamate transients were recorded in organotypic slices with bath application of $1.5 \mathrm{mM}$ DNI-Glutamate on the Femto3D-RC imaging system (both Femtonics, Budapest) using protocols as described above. Spot uncaging was accomplished using the single galvo scanner pair by rapid switching from the spiral scan imaging modality to a point scan located off the edge of the pre-synaptic bouton of interest for $0.2 \mathrm{~ms}$. During this time an uncaging pulse $\left(\lambda_{\mathrm{x}}{ }^{2 \mathrm{P}}=720 \mathrm{~nm}, 10-18 \mathrm{~mW}\right.$ depending on the depth of the bouton) was initiated by a $200 \mu \mathrm{s}$ square wave voltage applied to a Pockels cell (Conoptics M302RM, Danbury, CT) in the light path of the uncaging laser. Once an uncaging-evoked glutamate transient was evoked, the duration and power of the pulse were calibrated with the aim to minimise the pulse width and best match the decay kinetics of action potential evoked glutamate transients whilst producing reliable responses (range of duration used 10-50 $\mu \mathrm{s}$ ). The same protocol was then repeated four times with an interval of $100 \mathrm{~ms}$, for between 12 and 25 trials to match that using for measuring action potential evoked synaptic release.

\section{Dual transduction in the barrel cortex}

All animal procedures were conducted in accordance with the European Commission Directive (86/609/EEC) and the United Kingdom Home Office (Scientific Procedures) Act (1986). Adult C57BL/6J mice, male and female, were transfected with two viral constructs, encoding Syn.jRGECO1a and GFAP.SF-iGluSnFR.A184S respectively. Mice were anaesthetised (isoflurane, maintenance at $1.5-2 \%$ ), prepared for aseptic surgery and secured in a stereotaxic frame. Upon confirmation of the absence of pedal withdrawal 
reflex, two craniotomies of approximately $0.4 \mathrm{~mm}$ diameter were performed over the right hemisphere using a high-speed hand drill (Proxxon, Föhren, Germany), at sites overlying the posterior medial nucleus of the thalamus (POm) and the barrel cortex (S1BF). The entire microinjection into the POm was completed prior to performing the second craniotomy over S1BF. Stereotactic coordinates for POm injections (jRECO1a) were -2.2 $\mathrm{mm}$ and $1.2 \mathrm{~mm}$ along the anteroposterior and mediolateral axes, respectively. Two injection boluses were delivered at 2.9 and $3.1 \mathrm{~mm}$ beneath the dural surface. For S1BF injections (iGluSnFR), the bolus coordinates were $-0.5 \mathrm{~mm}$ and $3.0 \mathrm{~mm}$ along the anteroposterior and mediolateral axes, respectively, delivered at a depth of $0.6 \mathrm{~mm}$ along an oblique approach angle to avoid tissue scarring in S1BF that might occlude the optical window. A warmed saline solution was applied to exposed cortical surface during the procedure.

Pressure injections of AAV9 hSyn.jRGECO1a (totalling $0.5 \times 10^{10}$ genomic copies in a volume not exceeding $200 \mathrm{~nL}$, initially supplied by Penn Vector Core, PA, USA; and later by Addgene, MA, USA) and AAV2/5.GFAP.iGluSnFR.A184S ( $0.1 \times 10^{10}$ genomic copies, in a volume not exceeding $200 \mathrm{~nL}$, kindly gifted by Prof. Loren Looger) were carried out using a glass micropipette at a rate of $1 \mathrm{~nL}$ sec-1, stereotactically guided to the POm and S1BF, respectively, as outlined above. Once delivery was completed, pipettes were left in place for 5 minutes before being retracted. The surgical wound was closed and the animal recovered in a heated chamber. Meloxicam (subcutaneous, $1 \mathrm{mg} \mathrm{kg}^{-1}$ ) was administered once daily for up to two days following surgery. Mice were subsequently prepared for cranial window implantation approximately 2 weeks later.

\section{Cranial window implantation}

Mice were anaesthetised, prepared for aseptic surgery and secured in a stereotaxic frame as before during the viral transduction procedure. A large portion of the scalp was removed to expose the right frontal and parietal bones of the skull, as well as the medial aspects of the left frontal and parietal bones. The right temporalis muscles were reflected laterally to expose the squamous suture, to facilitate cement bonding during fixation of the cranial window implant. The exposed skull was coated with Vetbond (3M, MN, USA) and a custom-made headplate was affixed over the S1BF. The assembly was then secured with dental cement (SuperBond, Sun Medical Co. Ltd., Japan). Once the bonding agents had cured, the animal was removed from the stereotaxic frame and it's headplate was secured in a custom-built head fixation frame. A craniotomy of approximately $4 \mathrm{~mm}$ diameter was carried out over the right somatosensory cortex, centred over the S1BF injection site. Immediately prior to removal of the skull flap, the surface was superfused with warmed aCSF (in mM; $125 \mathrm{NaCl}, 2.5 \mathrm{KCl}, 26 \mathrm{NaHCO} 3,1.25 \mathrm{Na} 2 \mathrm{HPO} 4,18 \mathrm{Glucose}, 2 \mathrm{CaCl}$, 2 MgSO4; saturated with 95\% O2 / 5\% CO2, pH 7.4). The dura was resected using a combination of $26 \mathrm{G}$ needles (pushed against a hard surface to introduce a curved profile), fine-tipped forceps (11252-40, Fine Science Tools, Germany) and $2.5 \mathrm{~mm}$ spring scissors (15000-08, Fine Science Tools, Germany), taking care not to penetrate to the pia mater. Once the dura was removed, a previously-prepared coverslip consisting of a $34 \mathrm{~mm}$ diameter round coverglass affixed beneath a $4 \mathrm{~mm}$ diameter round coverglass (Harvard Apparatus UK, affixed using a UV-curable optical adhesive (NOA61), ThorLabs Inc., NJ, 
USA) was placed over the exposed cortex. Slight downward pressure was applied to the coverslip using a stereotactically guided wooden spatula that was previously severed and sanded to allow some flexibility and preclude excessive force, as previously described (80). The superfusion was discontinued and excess aCSF was removed using a sterile surgical sponge, taking care not to wick fluid from beneath the cranial window. The coverslip was then secured with VetBond and dental cement, sequentially. Once cured, the animal was recovered in a heated chamber and returned to its homecage when ambulatory. Post-operative care was administered as before during the viral transduction procedure. Animals were allowed habituate to headplate implantation within their homecage for at least one week.

\section{Multiplexed 2PE imaging in vivo}

Two-photon excitation was carried out using a wavelength multiplexing suite consisting of a Newport-Spectraphysics Ti:sapphire MaiTai tunable IR laser pulsing at $80 \mathrm{MHz}$ and a Newport-Spectraphysics HighQ-2 fixed-wavelength IR laser pulsing at $63 \mathrm{MHz}$, as detailed earlier $(36,68,81)$. The laser lightpaths were aligned (though not synchronised) before being point-scanned using an Olympus FV1000 with XLPlan N 25x water immersion objective (NA 1.05). Imaging was performed with head-fixed, awake animals as well as under lightly anaesthesia (fentanyl, $0.03 \mathrm{mg} \mathrm{kg}^{-1}$, midazolam, $3 \mathrm{mg} \mathrm{kg}^{-1}$, and medetomidine, $0.3 \mathrm{mg} \mathrm{kg}^{-1}$ ), with animals secured under the objective on a custom-built stage via the previously affixed headplate. First, exploratory acquisitions were performed with both lasers illuminating the tissue at $920 \mathrm{~nm}$ and $1045 \mathrm{~nm}$, respectively, in order to locate thalamocortical axons in S1BF ramifying within the arbor of iGluSnFR-positive cortical astrocytes. Brief pulses of nitrogen were directed at the contralateral whiskers to confirm tactile responses within the regions of interest (rhythmic whisker stimulation, RWS; 5 seconds, $3 \mathrm{~Hz}$ ). Measurements were performed throughout in L1 and L2/3, at depths of $50-150 \mathrm{~nm}$. For high resolution axonal bouton sampling, an initial framescan of $4-20 \mathrm{~Hz}$ was performed, with a pixel dwell time of $2 \mu \mathrm{s}$ and a mean laser power not greater than 30 $\mathrm{mW}$ at the focal plane. At this stage, tactile responses to short sensory stimulus trains (20 $\mathrm{Hz}, 200$ milliseconds) were monitored at axonal boutons, both before and after potentiation while in Tornado scanning mode $(400-500 \mathrm{~Hz})$. Sensory-evoked synaptic potentiation (RWS-LTP) within the barrel cortex was induced as previously described $(28,40)$, via a sustained contralateral rhythmic whisker stimulation (120 sec, $3 \mathrm{~Hz}$ ).

\section{Monte Carlo simulations of Brownian diffusion and cell surface binding}

Monte Carlo algorithms for particle diffusion were designed and run with MATLAB: there were previously described in detail, and tested and constrained using various experimental settings $(62,64,82)$. The simulation arena was a $4 \mu \mathrm{m}$ wide cube, with 1000 particles 'released' instantaneously at the centre. For each value of free parameter $\Psi$ (see below), 10 simulation trials were run, with different sphere distribution (generated so that $\beta=0.8 \pm$ 0.025 over the trials). Particles positioned at time $t$ at point $\mathbf{r}_{i}(x, y, z)$ were moved, over time step $\Delta t$, to point $\mathbf{r}_{i+1}\left(x+2 \delta_{x} \Delta_{1 \mathrm{D}}, y+2 \delta_{y} \Delta_{1 \mathrm{D}}, z+2 \delta_{z} \Delta_{1 \mathrm{D}}\right)$ where $\Delta_{1 \mathrm{D}}$ stands for the mean square displacement in the Einstein's diffusion equation for 1D Brownian motion $\Delta_{1 \mathrm{D}}^{2}=2 D \Delta t, D=0.65 \mu \mathrm{m}^{2} / \mathrm{ms}$ is the glutamate diffusion coefficient in the interstitial space 
(83), and $\delta_{x|y| z}$ denotes a 'delta-correlated' (independently seeded, uncorrelated) uniform random number from the $(-1,1)$ range. The latter ensures that Brownian particles are equally likely to move into either direction whereas scale factor 2 for $\delta$ gives the average elementary displacement in $x-y-z$ either $-\Delta_{1 \mathrm{D}}$ or $+\Delta_{1 \mathrm{D}}$. This algorithm provided the dutycycle translational particle movements in a contiguous 3D space, over all directions with varied 3D steps, rather than over the rectangular 3D-lattice vertices used by us and many others previously. The randomness of the displacement vector helped avoid occasional numerical deadlocks for particles trapped near the space dead-ends formed by aggregated overlapped spheres. The time step $\Delta t$ (usually $<0.1 \mu \mathrm{s}$ ) was set to be small enough to prevent particles from 'tunnelling' through the smallest, $50 \mathrm{~nm}$ wide obstacles, and the actual value of $D$ was verified at regular intervals.

The interaction with spheroids that represented either neuronal or astroglial fragments fell into the two corresponding cases. In the case of 'neuronal' spheroids, the interaction with diffusing glutamate particles was simulated as an elastic collision. In the case of 'astroglial' spheroids (which surface was populated with high-affinity transporters and/or iGuSnFR molecules), interaction was simulated as binding that occurred with the probability $P$. For each particle, $P$ was a function of time $t$ lapsed from its first collision with the current 'astroglial' spheroid, in accord with the basic lifetime expression for first-order reactions, $P=1-\exp \left(t \Psi^{-1}\right)$ where $\Psi$ is the time constant (free parameter) that determines how likely is the oncoming binding event. Parameter $\Psi$ thus combined, as a single quantity, the effects of binding affinity, binding-site surface density, and proximity to the diffusate. In practice, for each particle, computing $P$ continued as long as the particle was within $5 \mathrm{~nm}$ of the 'astroglial' spheroid; it was reset to zero when it was further away (increasing the cut-off distance above $5 \mathrm{~nm}$ had negligible effect on $P$ ). Once bound, the particle remained bound to astroglial spheroids for the simulated time period (4-5 ms) because the characteristic time of glutamate unbinding for either GLT-1 or iGluSnFR is much longer (tens of milliseconds).

\section{Simulating sphere-filled space representing barrel cortex neuropil}

There were at least two reasons to believe that randomly sized overlapping spheres would be a more realistic representation of neuropil compared to regular lattices of regular shapes, a tissue model used extensively by us and others previously. Firstly, multiple intersecting spheres give randomly shaped and randomly sized cellular elements and extracellular channels, as opposed to uniform or regular structures. Secondly, this approach provides a mixture of concave and convex shapes, including 'diffusion deadends' which are considered an important trait of brain neuropil (84). These realistic features of brain neuropil are not present in tissue models based on regular lattices.

Filling the space with the overlapping spheres followed the routines described in detail previously (64). In brief, the key parameter controlling this procedure was the volume fraction $\beta$ occupied by the spheres: $\beta=1-\alpha$ where $\alpha$ commonly stands for medium porosity, such as the volume fraction of the extracellular space in brain tissue. The $\beta$ value was calculated by (a) scattering $10^{5}$ test points uniformly randomly throughout the arena, and (b) calculating the proportion of the point falling outside the spheres. We verified that 
increasing the number of such test points to $10^{6}$ altered $\beta$ by $<1 \%$, pointing to asymptotic accuracy.

To fill the space with overlapping spheres that have a distributed size, we generated random $3 \mathrm{D}$ co-ordinates of sphere centroids across the simulation arena, and the random radius value for each sphere. The latter followed a uniform distribution between 50 and $300 \mathrm{~nm}$, which roughly represented characteristic widths of cortical neuropil elements (fragments of neuronal and astroglial processes) seen in electron micrographs. For any new computation, the initial number of spheres was estimated and distributed based on their average volume and the average size (to give the required $\beta=0.8$ value), and we left the co-ordinate origin unoccupied by any sphere. The space-filling cycle was repeated, with adjusted sphere numbers, until $\beta$ approached the required value with $\sim 5 \%$ accuracy.

\section{Computing environment}

Monte Carlo simulations were run using three computing environments. Firstly, a dedicated 8-node BEOWULF-style diskless PC cluster running under the Gentoo LINUX operating system (kernel 4.12.12), an upgraded version of that described earlier (85). Individual nodes comprised an HP ProLiant DL120 G6 Server containing a quad-core Intel Xeon X3430 processor and 8GB of DDR3 RAM. Nodes were connected through a NetGear Gigabit Ethernet switch to a master computer that distributes programs and collects the results on its hard disk. Secondly, on a UCL Myriad cluster: processors for each node, Intel(R) Xeon(R) Gold 6240 CPU @ 2.60GHz; cores per node $36+4$ A100 GPUs; RAM per node 192GB, tmpfs 1500G, total 6 nodes. Thirdly, cloud computing with Amazon AWS: t4g.medium, memory 4GB. Parallelisation and optimisation of the algorithms and program codes were implemented by AMC Bridge LLC (Waltham, MA). 


\section{References}

1. D. O. Hebb, The Organization of Behavior. (John Wiley \& Sons, New York, 1949).

2. T. Bliss, T. Lomo, Long-lasting potentiation of synaptic transmission in the dentate area of the anaesthetized rabbit following stimulation of the perforant path. J Physiol 232, 331-356 (1973).

3. A. Sale et al., Visual perceptual learning induces long-term potentiation in the visual cortex. Neurosci. 172, 219-225 (2011).

4. J. R. Whitlock, A. J. Heynen, M. G. Shuler, M. F. Bear, Learning induces long-term potentiation in the hippocampus. Science 313, 1093-1097 (2006).

5. E. Pastalkova et al., Storage of spatial information by the maintenance mechanism of LTP. Science 313, 1141-1144 (2006).

6. M. S. Rioult-Pedotti, D. Friedman, J. P. Donoghue, Learning-induced LTP in neocortex. Science 290, 533-536 (2000).

7. T. J. Ryan, D. S. Roy, M. Pignatelli, A. Arons, S. Tonegawa, Memory. Engram cells retain memory under retrograde amnesia. Science 348, 1007-1013 (2015).

8. G. G. Turrigiano, K. R. Leslie, N. S. Desai, L. C. Rutherford, S. B. Nelson, Activitydependent scaling of quantal amplitude in neocortical neurons. Nature 391, 892896 (1998).

9. L. F. Abbott, S. B. Nelson, Synaptic plasticity: taming the beast. Nat Neurosci 3 Suppl, 1178-1183 (2000).

10. K. Ibata, Q. Sun, G. G. Turrigiano, Rapid synaptic scaling induced by changes in postsynaptic firing. Neuron 57, 819-826 (2008).

11. H. K. Titley, N. Brunel, C. Hansel, Toward a Neurocentric View of Learning. Neuron 95, 19-32 (2017).

12. J. F. Disterhoft, M. M. Oh, Learning, aging and intrinsic neuronal plasticity. Trends Neurosci 29, 587-599 (2006).

13. D. Debanne, Information processing in the axon. Nature Rev. Neurosci. 5, 304-316 (2004).

14. J. Lisman, K. Cooper, M. Sehgal, A. J. Silva, Memory formation depends on both synapse-specific modifications of synaptic strength and cell-specific increases in excitability. Nature Neurosci. 21, 309-314 (2018).

15. W. C. Abraham, O. D. Jones, D. L. Glanzman, Is plasticity of synapses the mechanism of long-term memory storage? Npj Sci Learn 4, (2019).

16. G. Turrigiano, Too Many Cooks? Intrinsic and Synaptic Homeostatic Mechanisms in Cortical Circuit Refinement. Annual Review of Neuroscience, Vol 34 34, 89-103 (2011).

17. T. P. Jensen et al., Multiplex imaging relates quantal glutamate release to presynaptic $\mathrm{Ca} 2+$ homeostasis at multiple synapses in situ. Nature Communications 10, 1414 (2019).

18. T. P. Jensen, O. Kopach, J. P. Reynolds, L. P. Savtchenko, D. A. Rusakov, Release probability increases towards distal dendrites boosting high-frequency signal transfer in the rodent hippocampus. Elife 10, 62588 (2021).

19. L. E. Dobrunz, C. F. Stevens, Heterogeneity of release probability, facilitation, and depletion at central synapses. Neuron 18, 995-1008 (1997).

20. J. G. G. Borst, The low synaptic release probability in vivo. Trends Neurosci. 33, 259-266 (2010).

21. M. V. Tsodyks, H. Markram, The neural code between neocortical pyramidal neurons depends on neurotransmitter release probability. Proc Natl Acad Sci U S A 94, 719-723 (1997). 
22. M. D. McDonnell, L. M. Ward, The benefits of noise in neural systems: bridging theory and experiment. Nature Rev. Neurosci. 12, 415-U489 (2011).

23. T. G. Oertner, B. L. Sabatini, E. A. Nimchinsky, K. Svoboda, Facilitation at single synapses probed with optical quantal analysis. Nature Neurosci. 5, 657-664 (2002).

24. N. J. Emptage, C. A. Reid, A. Fine, T. V. Bliss, Optical quantal analysis reveals a presynaptic component of LTP at hippocampal Schaffer-associational synapses. Neuron 38, 797-804 (2003).

25. T. P. Jensen, K. Zheng, O. Tyurikova, J. P. Reynolds, D. A. Rusakov, Monitoring single-synapse glutamate release and presynaptic calcium concentration in organised brain tissue. Cell Calcium 64, 102-108 (2017).

26. P. Megevand et al., Long-term plasticity in mouse sensorimotor circuits after rhythmic whisker stimulation. J Neurosci 29, 5326-5335 (2009).

27. S. An, J. W. Yang, H. Sun, W. Kilb, H. J. Luhmann, Long-term potentiation in the neonatal rat barrel cortex in vivo. J Neurosci 32, 9511-9516 (2012).

28. F. Gambino et al., Sensory-evoked LTP driven by dendritic plateau potentials in vivo. Nature 515, 116-119 (2014).

29. K. Y. Zheng, T. P. Jensen, D. A. Rusakov, Monitoring intracellular nanomolar calcium using fluorescence lifetime imaging. Nature Protocols 13, 581-597 (2018).

30. T. Deneux et al., Accurate spike estimation from noisy calcium signals for ultrafast three-dimensional imaging of large neuronal populations in vivo. Nat Commun 7, 12190 (2016).

31. T. C. Sudhof, Calcium control of neurotransmitter release. Cold Spring Harb Perspect Biol 4, a011353 (2012).

32. H. Alle, J. R. Geiger, Combined analog and action potential coding in hippocampal mossy fibers. Science 311, 1290-1293 (2006).

33. R. Scott, A. Ruiz, C. Henneberger, D. M. Kullmann, D. A. Rusakov, Analog modulation of mossy fiber transmission is uncoupled from changes in presynaptic Ca2+. J. Neurosci. 28, 7765-7773 (2008).

34. J. Aguilar, M. L. Morales-Botello, G. Foffani, Tactile responses of hindpaw, forepaw and whisker neurons in the thalamic ventrobasal complex of anesthetized rats. Eur J Neurosci 27, 378-387 (2008).

35. J. F. Staiger, C. C. H. Petersen, Neuronal Circuits in Barrel Cortex for Whisker Sensory Perception. Physiol Rev 101, 353-415 (2021).

36. J. P. Reynolds, K. Zheng, D. A. Rusakov, Multiplexed calcium imaging of singlesynapse activity and astroglial responses in the intact brain. Neurosci Lett 689, 2632 (2019).

37. S. Ohno et al., A morphological analysis of thalamocortical axon fibers of rat posterior thalamic nuclei: a single neuron tracing study with viral vectors. Cereb Cortex 22, 2840-2857 (2012).

38. W. Zhang, R. M. Bruno, High-order thalamic inputs to primary somatosensory cortex are stronger and longer lasting than cortical inputs. Elife 8, (2019).

39. H. Dana et al., Sensitive red protein calcium indicators for imaging neural activity. Elife 5, (2016).

40. C. Henneberger et al., LTP Induction Boosts Glutamate Spillover by Driving Withdrawal of Perisynaptic Astroglia. Neuron 108, 919-936 e911 (2020).

41. M. Pignatelli et al., Engram Cell Excitability State Determines the Efficacy of Memory Retrieval. Neuron 101, 274-284 e275 (2019).

42. D. Debanne, M. M. Poo, Spike-timing dependent plasticity beyond synapse - preand post-synaptic plasticity of intrinsic neuronal excitability. Front Synaptic Neurosci 2, 21 (2010). 
43. J. F. Brons, C. D. Woody, Long-term changes in excitability of cortical neurons after Pavlovian conditioning and extinction. J Neurophysiol 44, 605-615 (1980).

44. A. P. Yiu et al., Neurons Are Recruited to a Memory Trace Based on Relative Neuronal Excitability Immediately before Training. Neuron 83, 722-735 (2014).

45. J. T. Paz et al., Multiple forms of activity-dependent intrinsic plasticity in layer $\mathrm{V}$ cortical neurones in vivo. J Physiol 587, 3189-3205 (2009).

46. S. Mahon, S. Charpier, Bidirectional plasticity of intrinsic excitability controls sensory inputs efficiency in layer 5 barrel cortex neurons in vivo. $J$ Neurosci 32, 11377-11389 (2012).

47. C. Gasselin, Y. Inglebert, D. Debanne, Homeostatic regulation of h-conductance controls intrinsic excitability and stabilizes the threshold for synaptic modification in CA1 neurons. J. Physiol. 593, 4855-4869 (2015).

48. I. van Welie, J. A. van Hooft, W. J. Wadman, Background activity regulates excitability of rat hippocampal CA1 pyramidal neurons by adaptation of a $\mathrm{K}+$ conductance. J Neurophysiol 95, 2007-2012 (2006).

49. P. J. Morgan, R. Bourboulou, C. Filippi, J. Koenig-Gambini, J. Epsztein, Kv1.1 contributes to a rapid homeostatic plasticity of intrinsic excitability in CA1 pyramidal neurons in vivo. Elife 8, (2019).

50. V. Sourdet, M. Russier, G. Daoudal, N. Ankri, D. Debanne, Long-term enhancement of neuronal excitability and temporal fidelity mediated by metabotropic glutamate receptor subtype 5. J Neurosci 23, 10238-10248 (2003).

51. S. Soldado-Magraner et al., Conditioning by subthreshold synaptic input changes the intrinsic firing pattern of CA3 hippocampal neurons. J Neurophysiol 123, 90-106 (2020).

52. C. D. Aizenman, D. J. Linden, Rapid, synaptically driven increases in the intrinsic excitability of cerebellar deep nuclear neurons. Nat Neurosci 3, 109-111 (2000).

53. O. Kopach, K. Y. Zheng, D. A. Rusakov, Optical monitoring of glutamate release at multiple synapses in situ detects changes following LTP induction. Molecular Brain 13, 39 (2020).

54. N. A. Lozovaya, M. V. Kopanitsa, Y. A. Boychuk, O. A. Krishtal, Enhancement of glutamate release uncovers spillover-mediated transmission by $\mathrm{N}$-methyl-Daspartate receptors in the rat hippocampus. Neurosci. 91, 1321-1330 (1999).

55. A. Scimemi, A. Fine, D. M. Kullmann, D. A. Rusakov, NR2B-containing receptors mediate cross talk among hippocampal synapses. J. Neurosci. 24, 4767-4777 (2004).

56. G. Szapiro, B. Barbour, Multiple climbing fibers signal to molecular layer interneurons exclusively via glutamate spillover. Nature Neurosci. 10, 735-742 (2007).

57. L. T. Coddington, S. Rudolph, P. Vande Lune, L. Overstreet-Wadiche, J. I. Wadiche, Spillover-Mediated Feedforward Inhibition Functionally Segregates Interneuron Activity. Neuron 78, 1050-1062 (2013).

58. E. A. Matthews et al., Optical Analysis of Glutamate Spread in the Neuropil. Cereb Cortex, (2022).

59. R. Bopp, S. Holler-Rickauer, K. A. Martin, G. F. Schuhknecht, An Ultrastructural Study of the Thalamic Input to Layer 4 of Primary Motor and Primary Somatosensory Cortex in the Mouse. J Neurosci 37, 2435-2448 (2017).

60. A. Motta et al., Dense connectomic reconstruction in layer 4 of the somatosensory cortex. Science 366, (2019).

61. D. A. Rusakov, D. M. Kullmann, M. G. Stewart, Hippocampal synapses: do they talk to their neighbours? Trends Neurosci 22, 382-388 (1999). 
62. L. P. Savtchenko, S. Sylantyev, D. A. Rusakov, Central synapses release a resource-efficient amount of glutamate. Nature Neurosci. 16, 10-U163 (2013).

63. K. Zheng, D. A. Rusakov, Efficient integration of synaptic events by NMDA receptors in three-dimensional neuropil. Biophys $J$ 108, 2457-2464 (2015).

64. L. P. Savtchenko, K. Zheng, D. A. Rusakov, Conductance of porous media depends on external electric fields. Biophys J 120, 1431-1442 (2021).

65. B. Zhou et al., Astroglial dysfunctions drive aberrant synaptogenesis and social behavioral deficits in mice with neonatal exposure to lengthy general anesthesia. PLoS Biol 17, e3000086 (2019).

66. E. Sykova, C. Nicholson, Diffusion in brain extracellular space. Physiol. Rev. 88, 1277-1340 (2008).

67. J. Tonnesen, V. V. G. K. Inavalli, U. V. Nagerl, Super-resolution imaging of the extracellular space in living brain tissue. Cell 172, 1108-1121 (2018).

68. K. Zheng et al., Time-resolved imaging reveals heterogeneous landscapes of nanomolar $\mathrm{Ca}^{2+}$ in neurons and astroglia. Neuron 88, 277-288 (2015).

69. K. P. Lehre, N. C. Danbolt, The number of glutamate transporter subtype molecules at glutamatergic synapses: Chemical and stereological quantification in young adult rat brain. J. Neurosci. 18, 8751-8757 (1998).

70. M. Armbruster, C. G. Dulla, J. S. Diamond, Effects of fluorescent glutamate indicators on neurotransmitter diffusion and uptake. Elife 9, (2020).

71. J. S. Diamond, C. E. Jahr, Transporters buffer synaptically released glutamate on a submillisecond time scale. J Neurosci 17, 4672-4687 (1997).

72. J. S. Marvin et al., Stability, affinity, and chromatic variants of the glutamate sensor iGluSnFR. Nature Methods 15, 936-939 (2018).

73. O. Tyurikova et al., $\mathrm{K}+$ efflux through postsynaptic NMDA receptors suppresses local astrocytic glutamate uptake. Glia, (2022).

74. A. C. Pereira et al., Glutamatergic regulation prevents hippocampal-dependent agerelated cognitive decline through dendritic spine clustering. Proc Natl Acad Sci U S A 111, 18733-18738 (2014).

75. A. Kruyer, M. D. Scofield, D. Wood, K. J. Reissner, P. W. Kalivas, Heroin CueEvoked Astrocytic Structural Plasticity at Nucleus Accumbens Synapses Inhibits Heroin Seeking. Biol Psychiat 86, 811-819 (2019).

76. L. Stoppini, P. A. Buchs, D. Muller, A simple method for organotypic cultures of nervous tissue. J. Neurosci. Meth. 37, 173-182 (1991).

77. D. A. Rusakov, A. Fine, Extracellular $\mathrm{Ca}^{2+}$ depletion contributes to fast activitydependent modulation of synaptic transmission in the brain. Neuron 37, 287-297 (2003).

78. R. Scott, D. A. Rusakov, Main determinants of presynaptic $\mathrm{Ca}^{2+}$ dynamics at individual mossy fiber-CA3 pyramidal cell synapses. J. Neurosci. 26, 7071-7081 (2006).

79. S. Rama et al., Presynaptic hyperpolarization induces a fast analogue modulation of spike-evoked transmission mediated by axonal sodium channels. Nat Commun 6, 10163 (2015).

80. G. J. Goldey et al., Removable cranial windows for long-term imaging in awake mice. Nature Protocols 9, 2515-2538 (2014).

81. A. Mishra et al., Astrocytes mediate neurovascular signaling to capillary pericytes but not to arterioles. Nature Neurosci 19, 1619-1627 (2016).

82. S. Sylantyev, L. P. Savtchenko, Y. Ermolyuk, P. Michaluk, D. A. Rusakov, SpikeDriven Glutamate Electrodiffusion Triggers Synaptic Potentiation via a HomerDependent mGluR-NMDAR Link. Neuron 77, 528-541 (2013). 
83. K. Y. Zheng et al., Nanoscale diffusion in the synaptic cleft and beyond measured with time-resolved fluorescence anisotropy imaging. Scientific Reports 7, 42022 (2017).

84. J. Hrabe, S. Hrabetova, K. Segeth, A model of effective diffusion and tortuosity in the extracellular space of the brain. Biophys J 87, 1606-1617 (2004).

85. K. Zheng, A. Scimemi, D. A. Rusakov, Receptor actions of synaptically released glutamate: the role of transporters on the scale from nanometers to microns. Biophys J 95, 4584-4596 (2008). 